\title{
Mediastinitis due to perforation by a metal stent after endoscopic ultrasound-guided hepaticogastrostomy: a rare complication
}

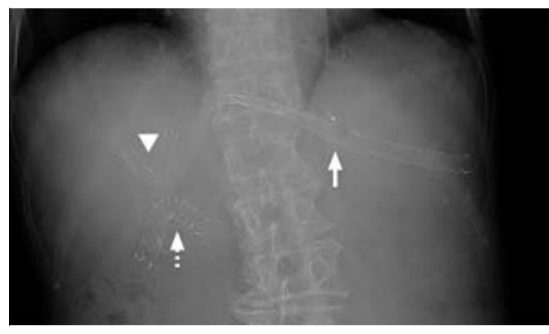

- Fig. 1 Radiographic image following endoscopic ultrasound-guided biliary drainage performed 3 months previously for hilar biliary obstruction due to recurrent pancreatic cancer after pancreaticoduodenectomy. Two metal stents were used in the procedure: one was inserted into the hilar biliary obstruction site to bridge the right and left hepatic ducts (arrowhead); the second was placed from intrahepatic bile duct segment 2 to the stomach (arrow). The length of the intragastric portion of the stent was about $7 \mathrm{~cm}$. The dotted arrow shows a metal stent placed for afferent loop syndrome.

Stents must be of a sufficient length to prevent their migration into the abdominal cavity after endoscopic ultrasoundguided hepaticogastrostomy (EUS-HGS) $[1,2]$. Although a self-expandable metal stent (SEMS) with a long intragastric portion can occasionally migrate to the esophagus, this hardly ever leads to severe complications. However, we present here a case of mediastinitis due to perforation caused by a SEMS.

A 75-year-old woman presented with intermittent vomiting for 2 days. She had undergone EUS-HGS 3 months previously for obstructive jaundice caused by post-resection recurrence of pancreatic

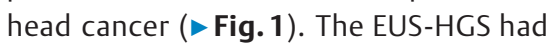
been performed from the stomach to intrahepatic bile duct segment 2 using a covered SEMS that was $8 \mathrm{~mm}$ in diameter and $12 \mathrm{~cm}$ in length (bare-end, Niti-S biliary S-type; Taewoong Corporation, Seoul, South Korea). A computed tomography $(C T)$ scan demonstrated that the SEMS had perforated the mediastinum beyond the digestive tract wall ( $>$ Fig. 2 ).
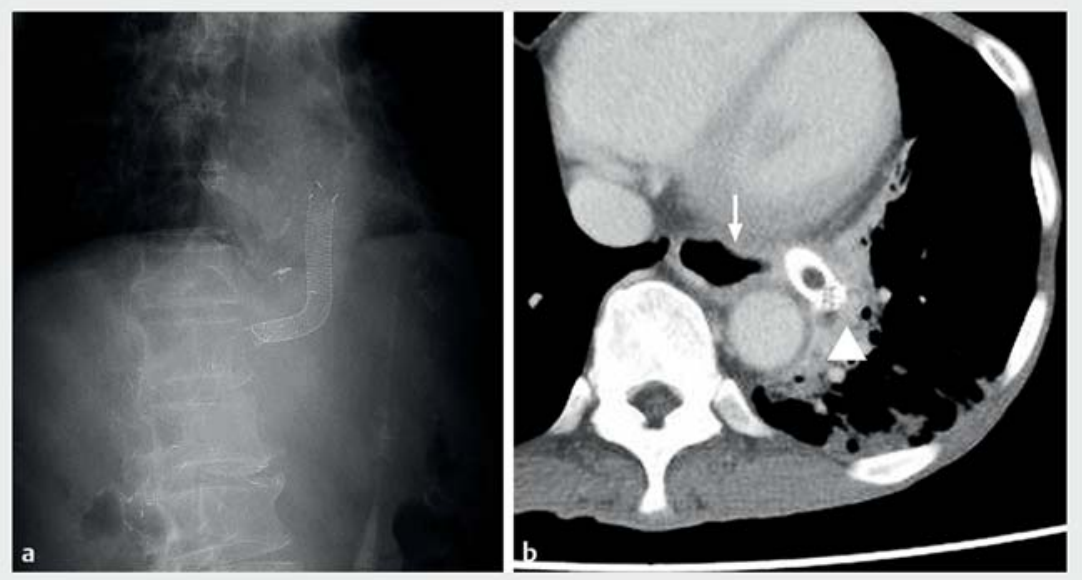

- Fig. 2 The migrated metal stent is revealed on: a a radiographic image showing an intragastric portion of the metal stent pointing upward, indicating its migration into the esophagus; $\mathbf{b}$ a computed tomography image showing a part of the stent penetrating the mediastinum (arrowhead). An arrow indicates the esophagus.
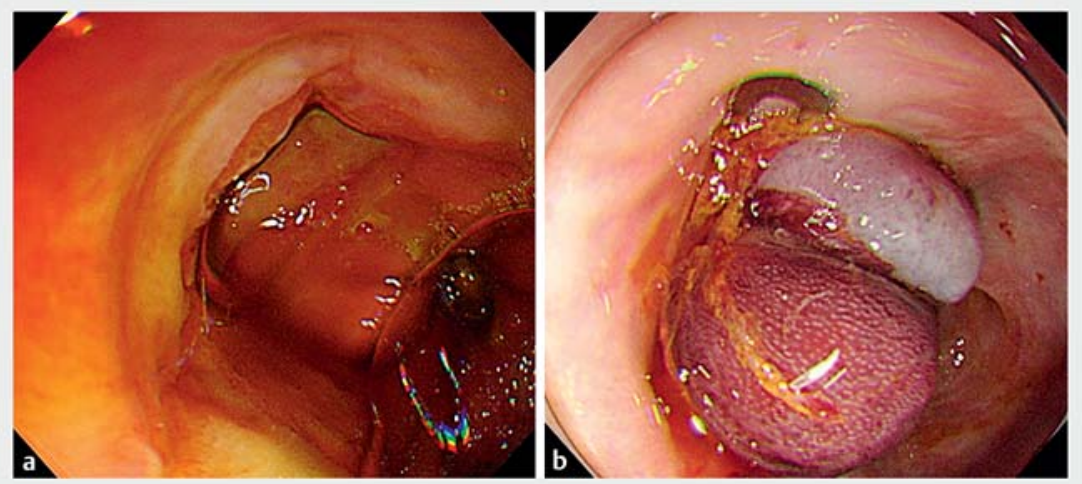

- Fig. 3 Endoscopic images showing: a the perforation site, which could be observed after the stent had been pushed back into the stomach; $\mathbf{b}$ the perforation site following its successful closure using an over-the-scope clip.

After introducing a gastroscope, we pushed the SEMS back into the stomach using biopsy forceps and identified the site of perforation ( $>$ Fig. 3 a; $>$ Video 1 ). We closed the perforation site using an over-the-scope clip (Ovesco Endoscopy GmbH, Tübingen, Germany) with the simple suction method because it was difficult to grasp both edges of the site using the dedicated forceps ( $\mathbf{F i g} \cdot \mathbf{3} \mathbf{b}$ ). Subsequently, argon plasma coagulation was used to trim an intragastric portion of the SEMS to prevent it penetrating the esophageal wall again. Radiographic imaging showed no extravasation of contrast medium and revealed closure of the perforation site 12 days later. The patient recovered well. 


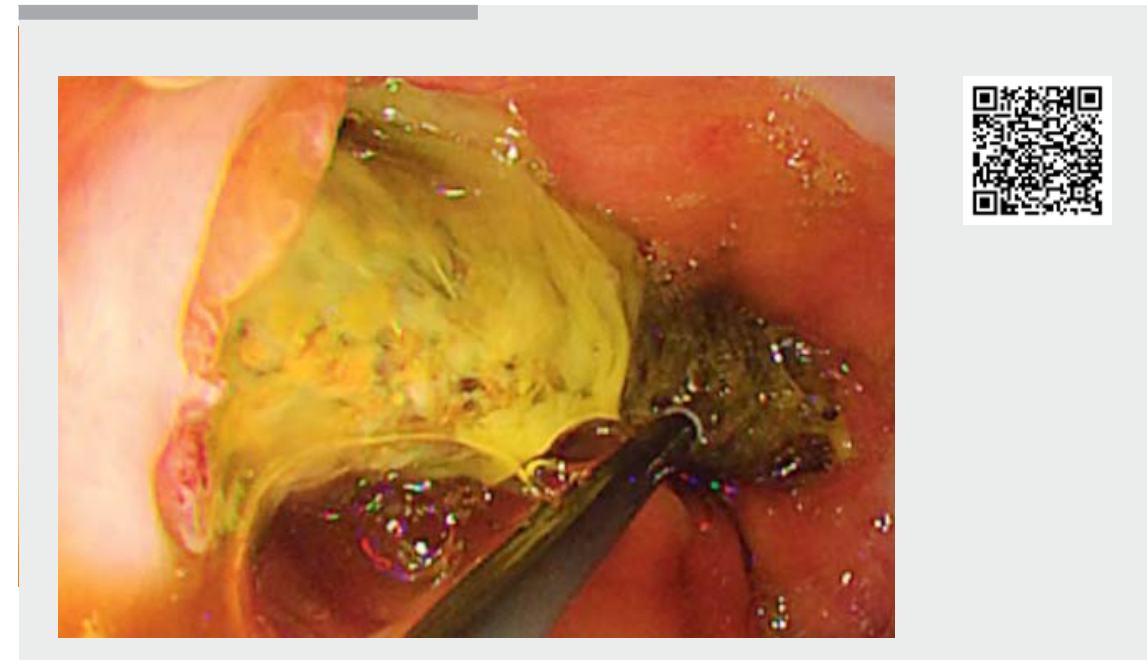

$\checkmark$ Video 1 We experienced a case of mediastinitis due to perforation by a long metal stent that had been placed during endoscopic ultrasound-guided hepaticogastrostomy 3 months previously and had migrated into the esophagus. This was managed by pushing the stent back into the stomach, clip closure of the perforation, and the subsequent shortening of the stent using argon plasma coagulation.
A long stent carries a possible risk of perforation and mediastinitis, as presented in our case; therefore, every endoscopist should consider this complication when using a long SEMS for EUS-HGS. Development of new SEMS designs is mandatory to avoid such complications in the future.

\section{The authors}

Junichi Kaneko' ${ }^{1}$, Hirotoshi Ishiwatari ${ }^{1}$, Kohei Takizawa ${ }^{1}$, Tatsunori Satoh ${ }^{1}$, Junya Sato ${ }^{1}$, Hiroyuki Matsubayashi ${ }^{1,2}$, Hiroyuki Ono ${ }^{1}$

1 Division of Endoscopy, Shizuoka Cancer Center, Shizuoka, Japan

2 Division of Genetic Medicine Promotion, Shizuoka Cancer Center, Shizuoka, Japan

\section{Corresponding author}

\section{Hirotoshi Ishiwatari, MD, PhD}

Division of Endoscopy, Shizuoka Cancer Center, 1007 Shimonagakubo Nagaizumicho, Sunto-gun, Shizuoka, Japan Fax: +81-55-9895551

ishihiro481019@gmail.com
Endoscopy_UCTN_Code_CPL_1AL_2AC

Competing interests

None 
Bibliography

[1] Isayama H, Nakai Y, Itoi T et al. Clinical practice guidelines for safe performance of endoscopic ultrasound/ultrasonographyguided biliary drainage: 2018. J Hepatobiliary Pancreat Sci 2019; 26: 249-269

[2] Nakai $\mathrm{Y}$, Isayama $\mathrm{H}$, Yamamoto $\mathrm{N}$ et al. Safety and effectiveness of a long, partially covered metal stent for endoscopic ultrasound-guided hepaticogastrostomy in patients with malignant biliary obstruction. Endoscopy 2016; 48: 1125-1128
DOI https://doi.org/10.1055/a-1065-1726

Published online: 9.12.2019

Endoscopy 2020; 52: 519-521

(c) Georg Thieme Verlag KG

Stuttgart · New York

ISSN 0013-726X

\section{ENDOSCOPY E-VIDEOS}

https:/|eref.thieme.de/e-videos

口回 Endoscopy E-Videos is a free access online section, reporting 靣: on interesting cases and new techniques in gastroenterological endoscopy. All papers include a high quality video and all contributions are freely accessible online.

This section has its own submission website at

https://mc.manuscriptcentral.com/e-videos 